activity during task performance. Consistent with their other results, the group found that, during the delay period, PPC neurons carried more information about the recent past than about the current tone held in working memory. Furthermore, across rats, the percentage of neurons holding such history-related information was tightly correlated with the level of history-induced bias in each animal's performance.

It has long been recognized that even simple perceptual decisions are not isolated, static computations - rather, they are dynamic processes embedded in a stream of past information $^{6}$. Akrami and colleagues have taken a crucial step towards understanding these processes, by identifying a node in the brain network that influences working-memory performance by holding a representation of recent history. That their discovery was made in an animal model offers the exciting possibility that future studies could investigate, at the cellular level, precisely which neurons in the PPC represent recent history, how this representation arises, and where and how it is integrated with sensory information to guide memory-based decision-making.

Statistical regularities not only influence working memory, but can also bias motor outputs and the encoding and decoding of sensory information ${ }^{7}$. It is therefore likely that history-related biases are implemented across many nodes of the decision-making brain network in addition to the PPC, through various mechanisms. Long-term mechanisms, on evolutionary and developmental scales, include the adaptation of neurons in sensory areas to the statistics of the natural environment. For example, the over-representation of vertical and horizontal contours in our visual environments is paralleled by an over-representation in the brain of neurons that respond preferentially to horizontal and vertical orientations ${ }^{8}$, and a biased ability to perceive these orientations ${ }^{9}$. Dynamic, short-term mechanisms occurring on a scale of milliseconds to seconds probably involve activity in long-range feedback or neuromodulatory circuits that adjust sensory areas ${ }^{10}$, the decision-making network ${ }^{11}$ and motor areas ${ }^{12}$. Elucidating the interplay between these mechanisms is just one of the remaining challenges in the quest to understand the powerful role of past experience in working memory.

\section{Laura Busse is in the Division of}

Neurobiology, Department Biology II,

LMU Munich, Munich 82152, Germany.

e-mail:busse@bio.lmu.de

1. Burton, G. J. \& Moorhead, I. R. Appl. Opt. 26, 157-170 (1987).

2. Dong, D. W. \& Atick, J. J. Netw. Comput. Neural Syst. 6, 345-358 (1995)

3. Akrami, A., Kopec, C. D., Diamond, M. E. \& Brody, C. D. Nature 554, 368-372 (2018)

4. Hollingworth, H. L. J. Phil. Psychol. Sci. Methods 7, 461-469 (1910)
5. Harvey, C. D., Coen, P. \& Tank, D. W. Nature 484, 62-68 (2012)

6. Gold, J. I. \& Stocker, A. A. Annu. Rev. Vis. Sci. 3, 227-250 (2017).

7. Kiyonaga, A., Scimeca, J. M., Bliss, D. P. \& Whitney, D. Trends Cogn. Sci. 21, 493-497 (2017)

8. Coppola, D. M., White, L. E., Fitzpatrick, D. \& Purves, D. Proc. Natl Acad. Sci. USA 95, 2621-2623 (1998)

9. Girshick, A. R., Landy, M. S. \& wSimoncelli, E. P.
Nature Neurosci. 14, 926-932 (2011).

10.St. John-Saaltink, E., Kok, P., Lau, H. C. \& de Lange, F. P. J. Neurosci. 36, 6186-6192 (2016).

11.Akaishi, R., Umeda, K., Nagase, A. \& Sakai, K. Neuron 81, 195-206 (2014).

12.Pape, A.-A. \& Siegel, M. Nature Commun. 7, 13098 (2016).

This article was published online on 7 February 2018

\title{
The two faces of a magnetic honeycomb
}

Quantum spin liquids are long-sought exotic states of matter that could transform quantum computing. Signatures of such a state have now been observed in a compound comprising iridium ions on a honeycomb lattice. SEE LETTER P.341

\section{MARTIN MOURIGAL}

$\mathrm{S}$ cientists are searching for elusive forms of magnetism in which spins - atomicscale 'compass needles' associated with electrons - perpetually dance to an intrinsic quantum beat. On page 341, Kitagawa et al. ${ }^{1}$ describe the synthesis and properties of a remarkable quantum magnet in which the ballet of spins persists down to a temperature of 0.05 kelvin. Such behaviour might be associated with exotic magnetic excitations that are of great fundamental interest and are sought for quantum-computing technologies.

Quantum magnets are often found in electrically insulating crystals. In such materials, unpaired electrons are arranged on a periodic lattice, which allows the spins of the electrons to interact with those of their neighbours. At low temperatures, these spins usually organize into symmetrical and regular patterns.

In rare cases, however, quantum fluctuations prevent the spins from becoming ordered. Instead, the spins enter a quantum superposition - a concerted and perpetual dance in which the spins are entangled, meaning that they are inseparable and share a common quantum state. Many flavours of such states, called quantum spin liquids, have been predicted $^{2}$. At first sight, these states resemble paramagnetic materials, in which spins are disordered in the absence of an external magnetic field. However, whereas spins behave as independent entities in paramagnets, those in quantum spin liquids are entangled with one another, even if separated by long distances.

Experimental physicists have long pondered how to obtain and detect quantum spin liquids in real materials ${ }^{3}$. Much has been learnt from the study of one-dimensional quantum magnets - in particular, from chains of spins that exhibit antiferromagnetism ${ }^{4}$, whereby each spin is aligned in the opposite direction to that of its neighbours. For instance, a by-product of long-range entanglement is the presence of magnetic excitations that have fractional quantum numbers (fractions of quantities such as electric charge and spin). These excitations have been shown to leave distinct fingerprints in measurements of a material's thermodynamic and magnetic properties. Furthermore, the presence or absence of an excitation gap (a lack of excitations that have particular energies) often reveals whether the underlying entanglement is short- or long-range, respectively.

However, in spite of these breakthroughs, finding two- and three-dimensional quantum spin liquids has been a daunting task ${ }^{5}$. One approach has been to use geometric frustration $^{3}$, in which there is an incompatibility between the spatial arrangement of spins and their interactions. This causes many spin configurations to have the same energy, which jump-starts entanglement. Kitagawa and colleagues used a different materials-science strategy, and focused on a quantum spin liquid that was proposed by the theoretical physicist Alexei Kitaev ${ }^{6}$ in 2006.

In Kitaev's model, spins on a honeycomb lattice are forced to interact in seemingly unnatural ways. The resulting quantum spin liquid has two types of exotic magnetic excitation: Majorana fermions, which have fractional quantum numbers, lack an excitation gap and can propagate on the lattice; and other excitations that have a small excitation gap and remain localized.

In a seminal paper ${ }^{7}$, it was demonstrated that the ingredients of Kitaev's model might exist in real materials, accompanied by more-conventional spin interactions called Heisenberg interactions. It was later suggested ${ }^{8}$ that the model could be realized in materials that have two key properties. The first is a strong coupling between the motion of electrons and their spin - a feature present in the 

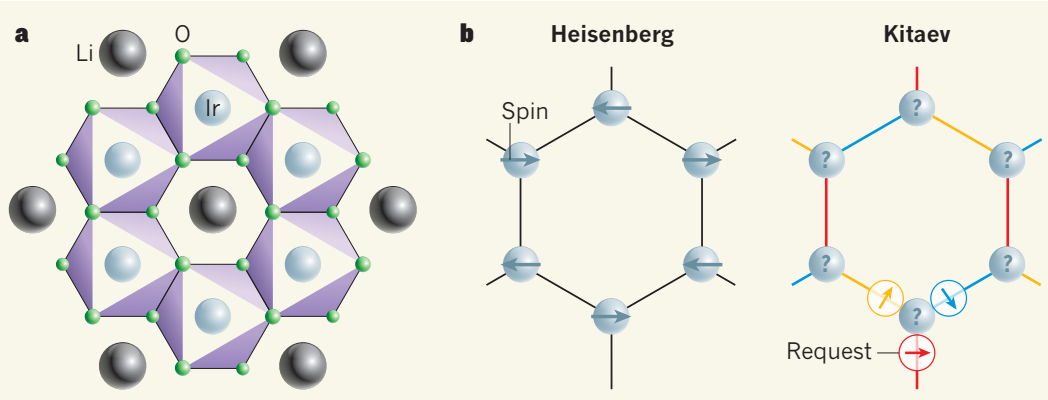

Figure 1 | The structure and spin interactions of $\mathrm{H}_{3} \mathrm{LiIr}_{2} \mathrm{O}_{6}$. a, Kitagawa et al. ${ }^{1}$ report that, unlike other magnetic materials, the compound $\mathrm{H}_{3} \mathrm{LiIr}_{2} \mathrm{O}_{6}$ does not show magnetic ordering at temperatures close to absolute zero ( $\mathrm{H}$, hydrogen; $\mathrm{Li}$, lithium; Ir, iridium; $\mathrm{O}$, oxygen). A single unit of a layer of the compound is shown here. Octahedra (purple) formed from iridium and oxygen ions cause the iridium ions to be arranged on a honeycomb lattice. $\mathbf{b}$, The magnetic properties of $\mathrm{H}_{3} \mathrm{LiIr}_{2} \mathrm{O}_{6}$ suggest that at least two types of interaction are at play between the spins (magnetic moments) of neighbouring iridium ions. The first are Heisenberg interactions (black lines), which cause spins to align in the opposite direction to that of their neighbours. The second are Kitaev interactions (coloured lines), which predispose neighbouring spins to co-align in one of three possible orientations, depending on which bond of the lattice is considered. Each line colour corresponds to a different preferred orientation. Because each lattice site participates in three distinct bonds, a given spin receives contradictory requests from its neighbours, which prevents magnetic ordering.

outer electron shells of some transition-metal ions. The second is a specific bonding geometry, which is achieved when octahedra formed from metal and oxygen ions share their edges to form a honeycomb lattice. Compounds such as lithium iridate ${ }^{9}\left(\mathrm{Li}_{2} \mathrm{IrO}_{3}\right)$, sodium iridate $\left(\mathrm{Na}_{2} \mathrm{IrO}_{3}\right)$ and ruthenium chloride ${ }^{10}\left(\mathrm{RuCl}_{3}\right)$ fulfil these requirements. However, all of these compounds become magnetically ordered below a few kelvin, which excludes them as genuine realizations of Kitaev's model.

Using an approach known as soft chemistry, in which materials are modified under mild temperature conditions (here, at $120^{\circ} \mathrm{C}$ ), Kitagawa et al. replaced inter-layer lithium ions of $\mathrm{Li}_{2} \mathrm{IrO}_{3}$ with hydrogen ions, while preserving the overall structure of the magnetic-honeycomb layers. The authors found that the resulting compound, $\mathrm{H}_{3} \mathrm{LiIr}_{2} \mathrm{O}_{6}$, has low-temperature properties that are spectacularly different from those of $\mathrm{Li}_{2} \mathrm{IrO}_{3}$. In particular, they observed no magnetic ordering in $\mathrm{H}_{3} \mathrm{LiIr}_{2} \mathrm{O}_{6}$ down to a temperature of $0.05 \mathrm{~K}$, and used nuclear magnetic resonance to confirm these findings down to $1 \mathrm{~K}$ (see Figure 2 of the paper ${ }^{1}$ ). The complex interplay between Kitaev and Heisenberg interactions ${ }^{11}$ is probably key to stabilizing this quantum state (Fig. 1). The authors discovered that the compound's thermodynamic and magnetic properties are highly unusual, and interpreted these measurements in terms of exotic magnetic excitations that lack an excitation gap and perhaps have fractional quantum numbers.

$\mathrm{H}_{3} \mathrm{LiIr}_{2} \mathrm{O}_{6}$ is a remarkable compound: it is the first iridium-based honeycomb magnet that does not become magnetically ordered at temperatures below a few kelvin. However, its overall behaviour and unusual thermodynamic properties indicate that it is governed by microscopic ingredients that differ substantially from those of Kitaev's model. This is not a curse, but a blessing: understanding exotic magnetic phases is often achieved by studying related materials and models ${ }^{12}$. Future experimental work in which, for example, large crystals of $\mathrm{H}_{3} \mathrm{LiIr}_{2} \mathrm{O}_{6}$ are grown, or particles such as neutrons or photons are scattered off $\mathrm{H}_{3} \mathrm{LiIr}_{2} \mathrm{O}_{6}$, could reveal whether the compound's excitations have fractional quantum numbers - the ultimate experimental proof of a quantum spin liquid.
The soft-chemistry approach used by Kitagawa et al. offers great promise for controlling the properties of layered quantum magnets. However, with regard to oxide materials, there are challenges associated with the presence of chemical disorder and heterogeneities. For instance, the layered structure of $\mathrm{H}_{3} \mathrm{LiIr}_{2} \mathrm{O}_{6}$ is prone to faults associated with the stacking of the layers ${ }^{13}$. In the future, it will be exciting to see chemists and physicists join forces to develop a deeper understanding of how material defects influence, and potentially even favour, entangled magnetic matter.

Martin Mourigal is in the School of Physics, Georgia Institute of Technology, Atlanta, Georgia 30332, USA.

e-mail:mourigal@gatech.edu

1. Kitagawa, K. et al. Nature 554, 341-345 (2018).

2. Savary, L. \& Balents, L. Rep. Prog. Phys. 80, 016502 (2016).

3. Imai, T. \& Lee, Y. Phys. Today 69, 30 (2016).

4. Lake, B. et al. Phys. Rev. Lett. 111, 137205 (2013).

5. Lee, P. A. Science 321, 1306-1307 (2008).

6. Kitaev, A. Ann. Phys. 321, 2-111 (2006).

7. Jackeli, G. \& Khaliullin, G. Phys. Rev. Lett. 102 017205 (2009).

8. Chaloupka, J., Jackeli, G. \& Khaliullin, G. Phys. Rev. Lett. 105, 027204 (2010)

9. Singh, Y. et al. Phys. Rev. Lett. 108, 127203 (2012). 10.Plumb, K. W. et al. Phys. Rev. B 90, 041112 (2014).

11.Rau, J. G., Lee, E. K. \& Kee, H. Y. Phys. Rev. Lett. 112, 077204 (2014).

12.Banerjee, A. et al. Nature Mater. 15, 733-740 (2016).

13.Bette, S. et al. Dalton Trans. 46, 15216-15227

(2017).

\section{MEDICAL RESEARCH}

\section{On the trail of invasive cells in breast cancer}

\section{During breast-cancer progression, tumour cells that arise in the milk duct spread elsewhere in the breast. The origin of these invasive tumour cells is now revealed by an analysis of spatially defined single cells.}

\section{DOUGLAS S. MICALIZZI \\ \& SHYAMALA MAHESWARAN}

I $\mathrm{n}$ the early-stage breast cancer called ductal carcinoma in situ (DCIS), a cluster of cancer cells arises in the milk duct and remains confined there. The recorded incidence of DCIS has risen since the late 1980s, probably due to an increase in its detection through more widespread breast-cancer screening ${ }^{1}$. Up to $40 \%$ of cases of DCIS progress to invasive ductal carcinoma (IDC), in which tumour cells invade other regions of the breast ${ }^{2-4}$. IDC requires clinical treatment. Moreover, because it is not possible to predict which people with DCIS are at risk of progressing to IDC, the standard treatment for patients who have DCIS is surgery, often followed by radiotherapy ${ }^{5}$. Therefore, understanding how DCIS progresses to IDC might enable the selective treatment of those at high risk of developing IDC. A paper in Cell by Casasent et al. ${ }^{6}$ reveals the origin of DCIS and its progression to IDC.

Casasent and colleagues obtained frozen samples of breast-cancer tissue gathered from patients. The authors used single-cell DNA sequencing of cells from DCIS and IDC regions in the same section of tissue to create molecular maps of patients' breast cancers, in an approach they call topographic single-cell sequencing. They stained the samples to identify tumour cells and noted the location of each selected cell in the regions of DCIS and IDC. A 